\title{
Effects of an irregular bedtime schedule on sleep quality, daytime sleepiness, and fatigue among university students in Taiwan Jiunn-Horng Kang ${ }^{1,2}$ and Shih-Ching Chen*2
}

\author{
Address: ${ }^{1}$ Sleep Science Center, Taipei Medical University Hospital, No. 252 Wu-Xing Street, Taipei 110, Taiwan, Republic of China and \\ 2Department of Physical Medicine and Rehabilitation, Taipei Medical University Hospital, No. 252 Wu-Xing Street, Taipei 110, Taiwan, Republic \\ of China \\ Email: Jiunn-Horng Kang - jhk@tmu.edu.tw; Shih-Ching Chen* - csc@tmu.edu.tw \\ * Corresponding author
}

Published: 19 July 2009

BMC Public Health 2009, 9:248 doi:10.1 | 86/ | 47/-2458-9-248

This article is available from: http://www.biomedcentral.com/I47/-2458/9/248

(C) 2009 Kang and Chen; licensee BioMed Central Ltd.

This is an Open Access article distributed under the terms of the Creative Commons Attribution License (http://creativecommons.org/licenses/by/2.0), which permits unrestricted use, distribution, and reproduction in any medium, provided the original work is properly cited.
Received: 14 January 2009

Accepted: 19 July 2009

\begin{abstract}
Background: An irregular bedtime schedule is a prevalent problem in young adults, and could be a factor detrimentally affecting sleep quality. The goal of the present study was to explore the association between an irregular bedtime schedule and sleep quality, daytime sleepiness, and fatigue among undergraduate students in Taiwan.

Methods: A total of 160 students underwent a semi-structured interview and completed a survey comprising 4 parts: Pittsburgh Sleep Quality Index (PSQI), Epworth Sleepiness Scale (ESS), Fatigue Severity Scale (FSS), and a rating of irregular bedtime frequency. Participants were grouped into 3 groups in terms of irregular bedtime frequency: low, intermediate, or high according to their 2week sleep log. To screen for psychological disorders or distress that may have affected responses on the sleep assessment measures, the Chinese health questionnaire-I2 (CHQ-I2) was also administered.

Results: We found an increase in bedtime schedule irregularity to be significantly associated with a decrease in average sleep time per day (Spearman $r=-0.22, p=0.05$ ). Multivariate regression analysis revealed that irregular bedtime frequency and average sleep time per day were correlated with PSQI scores, but not with ESS or FSS scores. A significant positive correlation between irregular bedtime frequency and PSQI scores was evident in the intermediate (partial $r=0.18, p=$ 0.02 ) and high (partial $r=0.15, p=0.05$ ) frequency groups as compared to low frequency group.

Conclusion: The results of our study suggest a high prevalence of both an irregular bedtime schedule and insufficient sleep among university students in Taiwan. Students with an irregular bedtime schedule may experience poor sleep quality. We suggest further research that explores the mechanisms involved in an irregular bedtime schedule and the effectiveness of interventions for improving this condition.
\end{abstract}

\section{Background}

Some behaviours or activities are detrimental to normal sleep have been suggested. These "inadequate sleep hygiene" behaviours include irregular sleep schedules, fre- quent or prolonged daytime naps, excessive alcohol consumption before bedtime, and staying on one's bed for non-sleep-related activities [1-3]. Accordingly, adequate sleep hygiene is considered to be an important adjuvant 
for treating patients with insomnia or other sleep disturbances $[1,3,4]$. However, in the case of normal subjects, who are unaffected by these pathological conditions, the association between sleep hygiene and sleep itself is surprisingly inconsistent [5-8].

Sleep is regulated by two main processes: the sleep homeostatic drive, influenced by experienced durations of sleep and wakefulness, and the circadian system, an intrinsic pacemaker involving a pathway from the suprachiasmatic nucleus to the hypothalamus [9]. The circadian system has complex interactions with daily behaviours, known as entraining factors. It is thought that having a regular bedtime schedule can strengthen the circadian rhythm; and is beneficial for achieving a good quality of sleep [10]. Even one-night alteration to a sleep schedule can be sufficient to induce difficulties with sleep initiation and maintenance. Taub et al. reported that an acute shift in sleep schedules of 2 hours without altering total duration of sleep can decrease cognitive and psychological functioning in a laboratory setting [11-14]. However, it is unclear if this finding can be generalised to real life conditions over long durations.

Another condition associated with frequent changes in the sleep-wake schedule is in the case of shift workers. Shift work is found to adversely affect sleep and general health, including decreased sleep quality, altered sympathetic activity, increased risk of cardiovascular events, and reduced cognitive performance [15-17]. However, the changing sleep/wake pattern of shift workers differs greatly from that of young adults; the phase shifts of the latter group are shorter but more irregular than the former. This suggests that the data obtained from shift workers may not adequately represent those for young adults with an irregular sleep schedule.

Although the prevalence varies, many adolescents and young adults are reported to have an irregular sleep schedule and a tendency to have a delayed sleep phase [18-22]. Furthermore, a remarkable degree of problems associated with sleeping and poor sleep quality have been observed in university students of many Western countries [18-22]. However, to our knowledge, the data regarding sleep patterns and habits in the Chinese is limited. It is necessary to investigate this issue within the Chinese population because sleep habits are affected by ethnicity, social factors, and culture $[20,22]$. Our aim was to investigate sleep quality and associated daytime effects in Chinese undergraduate students. We were particularly interested in determining how bedtime schedule relates to sleep quality and daytime functioning.

\section{Methods \\ Sampling and study population}

The Taipei Medical University Hospital Review Board approved the present study, and all participants provided informed consent. The participants were selected randomly from first-year undergraduate students from a medical university in Taipei, Taiwan. We excluded subjects with a history of either chronic medical or psychotic disorders, as well as those currently on medication. Of 197 students interviewed, 160 (81.2\%) completed the survey; 81 males and 79 females, with a mean age of 20.3 \pm 1.9 years. were included in the study.

\section{Measures and data management}

A flowchart of the survey and data collection process is presented in Figure 1. Participants were first asked to maintain a prospective 2 -week sleep log. Subsequently, they underwent a semi-structured interview, and the quality of entries in their sleep log was reviewed at that time. Sleep schedule and average daily sleep time data were obtained and analyzed from the sleep logs. To decrease the difficulty and uncertainty of scoring, irregular bedtime frequency was defined as the number of nights when a greater than 1 hour shift in bedtime from the usual bed-

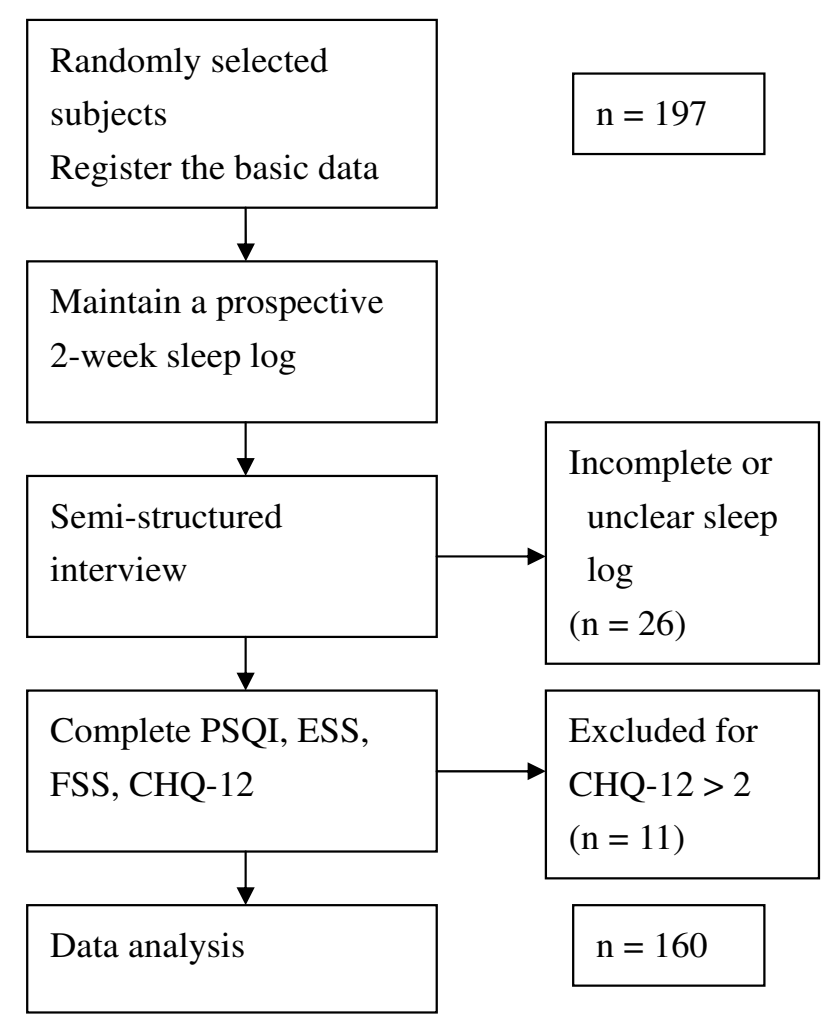

Figure I Flowchart of the survey and data collection process. 
time had occurred in the past 2 weeks. The subjects were assigned to 1 of 3 irregular bedtime frequency groups: low, $\leq 1$ night per week; intermediate, 1-3 nights per week; and high, $\geq 3$ nights per week or not having a regular bedtime.

The interview involved the completion of 3 questionnaires evaluating sleep quality, daytime sleepiness, and fatigue. Sleep quality was assessed using the Pittsburgh Sleep Quality Index (PSQI), which is widely accepted as both valid and reliable $[23,24]$. The PSQI includes 19 items, and yields a score from 0 (good quality) to 21 (poor quality). Sleep onset latency and sleep efficiency, defined as the actual sleep time divided by the time in bed, are also obtained with the PSQI. Daytime sleepiness was assessed using the Epworth Sleepiness Scale (ESS), a widely used and reliable predictor of daytime sleepiness [25]. The ESS employs a 4-point scale to rank the chances of a subject falling asleep in different scenarios. Fatigue was evaluated using the Fatigue Severity Scale (FSS); this scale includes 9 questions, yields a total score from 9 (no fatigue) to 63 (severe fatigue), and has been previously applied in clinical evaluations of fatigue $[26,27]$.

Subjects were also administered the CHQ-12 (Chinese health questionnaire-12) in order to screen for psychological disorders or distresses that may have affected responses on the sleep assessment measures. The CHQ-12 includes 12 items, and has been shown as valid for use with the Chinese population [28]. We excluded 11 students from the study for having a CHQ-12 score greater than 2 points.

\section{Statistical analysis}

There were five major dependent variables: sleep onset latency, sleep efficiency, and scores for each of the PSQI, ESS, and FSS. Student's $t$-test was used to compare males and females with respect to each variable. Chi-square test and Spearman's correlation were used to analyse relationships between irregular bedtime frequency and average sleep time per day.
To control for the potential confounding effects of sleep insufficiency, multivariate linear regression adjusted for average daily sleep time was used to analyse how irregular bedtime frequency related to scores for each of the PSQI, ESS, and FSS. The analysis was performed by setting the intermediate and high FIB groups as dummy variables in comparison to the low FIB group. The significance level was set as $\mathrm{p}<0.05$.

\section{Results}

There was no statistical difference between males and females for any variable; therefore, the data for both genders was combined in all subsequent analyses. Across all subjects, the mean sleep onset latency was $14.2 \pm 10.6$ min, and the mean average daily sleep time was $6.7 \pm 1.3$ h. Mean scores for the FSS, ESS, and PSQI were 38.2 \pm 8.9 , $6.3 \pm 3.3$, and $4.9 \pm 2.4$, respectively. The data for all variables and subjects are summarised in Table 1. Poor sleep quality (PSQI score $>5$ ), daytime sleepiness (ESS score $>10$ ), and fatigue (FSS score $\geq 36$ ) were each reported by a considerable proportion of participants, $33.8 \%, 14.4 \%$, and $37.5 \%$, respectively.

With respect to irregular bedtime frequency, $26.9 \%$ of the participants were in the low frequency group $(<1$ night per week), $38.8 \%$ were in the intermediate frequency group (1-3 nights per week), and $34.4 \%$ were in the high frequency group ( $>3$ nights per week). Nearly half of all subjects $(46.9 \%)$ reported an average daily sleep time that was less than $7 \mathrm{~h}$. It is worth noting that average daily sleep time was negatively correlated with irregular bedtime frequency $(\mathrm{r}=-0.22, \mathrm{p}<0.05)$. The distribution of average daily sleep time across irregular bedtime frequency groups is shown in Table 2.

Multivariate regression analysis revealed irregular bedtime frequency and average daily sleep time had a significant positive correlation with PSQI scores $(\mathrm{r}=0.61$, $\mathrm{p}<$ 0.001 ), but not with ESS or FSS scores. With adjustment for average daily sleep time, this positive correlation of irregular bedtime frequency with PSQI scores was more evident in the intermediate (partial $r=0.18, p=0.02$ ) and

Table I: Mean (standard deviation (SD)) results of all measures for both genders and all subjects

\begin{tabular}{llll}
\hline Variable & Female $(\mathbf{n}=\mathbf{8 I})$ & Male $(\mathbf{n}=\mathbf{7 9})$ & Total $(\mathbf{n}=\mathbf{1 6 0})$ \\
\hline SOL (min) & $15.2(11.3)$ & $12.9(9.8)$ & $14.2(10.6)$ \\
SE (\%) & $89.0(14.4)$ & $91.1(10.5)$ & $90.1(12.6)$ \\
ADST (h) & $6.8(1.4)$ & $6.6(1.1)$ & $6.7(1.3)$ \\
ESS score & $6.7(3.2)$ & $5.9(3.4)$ & $6.3(3.3)$ \\
FSS score & $38.0(8.5)$ & $38.5(9.4)$ & $38.2(8.9)$ \\
PSQl score & $5.0(2.4)$ & $4.8(2.3)$ & $4.9(2.4)$ \\
\hline
\end{tabular}

SOL, sleep onset latency; SE, sleep efficiency; ADST, average daily sleep time; ESS, Epworth Sleepiness Scale; FSS, Fatigue Severity Scale; PSQI, Pittsburgh Sleep Quality Index

There was no significant difference $(p<0.05)$ between genders for any variable 
Table 2: Number (\%) of subjects in each irregular bedtime frequency group with an average daily sleep time $<7 \mathrm{~h} /$ day, $7-8 \mathrm{~h} /$ day, or $>8$ h/day

\begin{tabular}{|c|c|c|c|c|}
\hline \multirow[t]{2}{*}{ ADST* } & \multicolumn{3}{|c|}{ Irregular bedtime frequency ${ }^{+}$} & \multirow[t]{2}{*}{ Total } \\
\hline & Low & Intermediate & High & \\
\hline$<7$ h/day & $14(8.8)$ & $26(16.3)$ & $35(21.9)$ & 75 (46.9) \\
\hline 7-8 h/day & $17(10.6)$ & $24(15.0)$ & $10(6.3)$ & $51(31.9)$ \\
\hline$>8 \mathrm{~h} / \mathrm{day}$ & $12(7.5)$ & $12(7.5)$ & $10(6.3)$ & $34(21.3)$ \\
\hline Total & $43(26.9)$ & $62(38.8)$ & $55(34.4)$ & $160(100)$ \\
\hline
\end{tabular}

ADST, average daily sleep time

+ An irregular bedtime event was defined as a shift from the usual bedtime of $>$ I h. This occurred $<\mid$ night/week for the low-frequency group, I-3 nights/week for the intermediate-frequency group, and $>3$ nights/week for the high-frequency group.

*Chi-Square analysis indicated that average daily sleep time differed significantly across the irregular bedtime frequency groups $\left(\chi^{2}=\right.$ I I.68, $p=0.022$ ); as supported by a significant Spearman correlation $(r=-0.22, p=0.05)$.

high (partial $r=0.15 ; p=0.05$ ) frequency groups than the low frequency group (Table 3 ).

\section{Discussion}

Our findings indicated that students with a frequently irregular bedtime had poor sleep quality, even after adjusting for the sleep time. We also found negative trends between an irregular bedtime schedule and daytime functioning; although those concerning ESS and FSS scores were not statistically significant. The consequences of an irregular bedtime schedule may be less significant than those of sleep insufficiency. However, the adverse

Table 3: Results of multivariate regression analysis of irregular bedtime frequency and average daily sleep time on PSQI, ESS, and FSS scores

\begin{tabular}{lrrrr}
\hline & & ESS & FSS & PSQI \\
\hline Intermediate FIB (p-value) & $(0.389)$ & $(0.132)$ & $\left(0.02^{*}\right)$ \\
& Zero-order r & -0.05 & 0.05 & 0.05 \\
& Partial r & 0.07 & 0.12 & 0.18 \\
High FIB (p-value) & & $\left(0.015^{*}\right)$ & $(0.124)$ & $\begin{array}{r}\left(0.05^{*}\right) \\
0.12\end{array}$ \\
& Zero-order r & 0.18 & 0.09 & 0.20 \\
& Partial r & 0.19 & 0.12 & 0.15 \\
ADST (p-value) & & $(0.314)$ & $(0.359)$ & $(<0.001 *)$ \\
& Zero-order r & 0.02 & -0.11 & -0.59 \\
& Partial r & 0.08 & -0.07 & 0.56 \\
\hline \multirow{2}{*}{ Total r (p-value) } & & 0.20 & 0.17 & 0.61 \\
& & $(0.09)$ & $(0.196)$ & $(<0.001 *)$ \\
\hline
\end{tabular}

FIB, frequency of irregular bedtime; ADST, average daily sleep time; ESS, Epworth Sleepiness Scale; FSS, Fatigue Severity Scale; PSQI,

Pittsburgh Sleep Quality Index; r, correlation coefficients.

The multivariate regression analysis was performed by setting the intermediate and high FIB groups as dummy variables in comparison to the low FIB group

$*_{p}<0.05$ effects of an irregular bedtime schedule on sleep should not be overlooked. We postulate that an irregular bedtime schedule affects sleep quality by disturbing the circadian rhythm. Previous studies have shown that disturbances of the circadian rhythm produce alterations in sleep architecture and sleep quality known to be associated with fatigue, vigilance problems, decreased productivity, and negative health effects $[10,29]$.

We found that students with a frequently irregular bedtime also had a relatively short average sleep time per day, which could put them at risk of sleep insufficiency. This finding may be partially explained by the daytime activity schedules (for classes or recreation) of these students limiting their ability to get enough sleep in the morning. In addition, environmental factors like light or noise can also be detrimental to people trying to sleep late into the morning or day. Many studies have reported that an accumulated sleep deprivation induces impairments of cognition, vigilance, and memory, and disturbances of mood [30-33]. We suggest that students with an irregular bedtime schedule should be encouraged to maintain a regular bedtime, and to increase their total sleep time. These changes might help to increase the sleep quality and daytime functioning of these students.

The mechanisms by which an irregular sleep schedule develops in young adults are not well understood; however, they are likely to be multi-factorial, including biological, behavioural, and social factors. Two endogenous circadian rhythm patterns of alertness and sleepiness have been observed in the general population, in accordance with which people may be classified as being of a morning type (M-type) or an evening type (E-type) [34]. The sleep/ wake schedule of these types differs, with the E-type associated with irregular sleeping habits and an increased need for sleep $[35,36]$. In addition to biological factors, social cues, activity types and levels, environmental factors, and motivation to maintain a regular bedtime all also play important roles in entraining the sleep-wake cycle by adjusting the intrinsic (endogenously regulated) circadian rhythm [10]. Further research is needed to clarify the underlying biological and behavioural mechanisms by which an irregular bedtime schedule develops.

Our results indicate that there is a high prevalence of both an irregular bedtime schedule and short average daily sleep time among undergraduate students in Taiwan. These findings are compatible with those of studies from other countries, thereby suggesting that poor sleep in university students may be a universal and prevalent problem of modern society $[18,19,21]$. In reviewing the research on sleep patterns in university students, Hicks et al. found the median sleep time to have dropped by about 1 hour over the past 20 years [37]. Lifestyle, social and academic 
schedules, and insufficient sleep education could all contribute to the aetiology of chronic sleep insufficiency and poor sleep in university students $[18,19,38]$. Some approaches to enhancing the sleep hygiene and relevant knowledge of university students have been developed, but the effectiveness of these remains to be confirmed [38].

The present study has some limitations. First, the selfreported estimation of average daily sleep time and irregular bedtime frequency may have been influenced by subjects' cooperation and a recall bias. An objective assessment method such as actigraphy is required for more accurate measurements of these parameters. Moreover, the university medical students we studied might not be representative of the more general population of university students in Taiwan. A randomised, populationbased study with a large sample size will be required to confirm our findings. A further limitation of our cross-sectional study is the difficultly in establishing causality. It is possible that subjects who have poor sleep quality (e.g., sleep onset difficulty or sleep fragmentation) could subsequently develop inadequate sleep habits, including an irregular sleep schedule. Longitudinal studies are thus required to clarify the cause-and-effect relationships between an irregular bedtime schedule and poor sleep quality. Finally, there are many different inadequate sleep habits and sleep-related behaviours likely to have a detrimental impact on sleep quality in normal subjects. An irregular bedtime schedule was the only one of these factors investigated in the present study, and future studies should take more of these factors into consideration.

\section{Conclusion}

Our results suggest that there is a high prevalence of both an irregular bedtime schedule and insufficient sleep among university students in Taiwan. Students with an irregular bedtime schedule may also experience poor sleep quality. Further research exploring the mechanisms involved in an irregular bedtime schedule and the effectiveness of interventions for improving this condition should be conducted.

\section{Competing interests}

The authors declare that they have no competing interests.

\section{Authors' contributions}

JHK participated in designing the study, conducting the interviews, performing data analysis, and drafting the manuscript. CSC participated in designing and coordinating the study. Both authors read and approved the final manuscript.

\section{Acknowledgements}

We sincerely thank Misses YG Huang, HI Su, and Tina Huang for their assistance in conducting the interviews and data management.

\section{References}

I. Hauri PJ: Advances in the behavioral treatment of insomnia. Sleep Med Rev 2003, 7:201-202.

2. Jefferson CD, Drake CL, Scofield HM, Myers E, McClure T, Roehrs T, Roth T: Sleep hygiene practices in a population-based sample of insomniacs. Sleep 2005, 28:6 I I-6I5.

3. Stepanski EJ, Wyatt JK: Use of sleep hygiene in the treatment of insomnia. Sleep Med Rev 2003, 7:2 I5-225.

4. Guedes BM, Soares V, Carlos WJ: Impact of sleep hygiene on patients with obstructive sleep apnoea syndrome. Rev Port Pneumol 2006, I 2: 147-I76.

5. Cheek RE, Shaver JL, Lentz MJ: Lifestyle practices and nocturnal sleep in midlife women with and without insomnia. Biol Res Nurs 2004, 6:46-58.

6. Cheek RE, Shaver JL, Lentz MJ: Variations in sleep hygiene practices of women with and without insomnia. Res Nurs Health 2004, 27:225-236.

7. LeBourgeois MK, Giannotti F, Cortesi F, Wolfson A, Harsh J: Sleep hygiene and sleep quality in Italian and American adolescents. Ann NY Acad Sci 2004, 102 I:352-354.

8. Manni R, Politini L, Ratti MT, Marchioni E, Sartori I, Galimberti CA, Tartara A: Sleep hygiene in adult epilepsy patients: a questionnaire-based survey. Acta Neurol Scand 2000, I 0 I:301-304.

9. Dijk DJ, Lockley SW: Integration of human sleep-wake regulation and circadian rhythmicity. J Appl Physiol 2002, 92:852-862.

10. Borbely AA, Achermann P: Sleep homeostasis and models of sleep regulation. J Biol Rhythms 1999, I4:557-568.

II. Taub JM: Effects of ad lib extended-delayed sleep on sensorimotor performance, memory and sleepiness in the young adult. Physiol Behav 1980, 25:77-87.

12. Taub JM, Berger RJ: Performance and mood following variations in the length and timing of sleep. Psychophysiology 1973, 10:559-570.

13. Taub JM, Berger RJ: Acute shifts in the sleep-wakefulness cycle: effects on performance and mood. Psychosom Med 1974, 36: $164-173$.

14. Taub JM, Berger RJ: The effects of changing the phase and duration of sleep. J Exp Psychol Hum Percept Perform 1976, 2:30-4I.

15. Kecklund G, Ekstedt M, Akerstedt T, Dahlgren A, Samuelson B: The effects of double-shifts ( 15.5 hours) on sleep, fatigue and health. J Hum Ergol (Tokyo) 200I, 30:53-58.

16. Phillips B, Magan L, Gerhardstein C, Cecil B: Shift work, sleep quality, and worker health: a study of police officers. South Med J I99I, 84: I I76-II84. II96

17. Shen J, Botly LC, Chung SA, Gibbs AL, Sabanadzovic S, Shapiro CM: Fatigue and shift work. J Sleep Res 2006, I 5: I-5.

18. Brown FC, Buboltz WC Jr, Soper B: Relationship of sleep hygiene awareness, sleep hygiene practices, and sleep quality in university students. Behav Med 2002, 28:33-38.

19. Buboltz WC Jr, Brown F, Soper B: Sleep habits and patterns of college students: a preliminary study. J Am Coll Health 200I, 50: | $3|-| 35$.

20. Hicks RA, Lucero-Gorman K, Bautista J, Hicks GJ: Ethnicity, Sleep Hygiene Knowledge, and Sleep Hygiene Practices. Percept Mot Skills 1999, 88:1095-1096.

21. Lack LC: Delayed sleep and sleep loss in university students. J Am Coll Health 1986, 35: I05-I I0.

22. Manni R, Ratti MT, Marchioni E, Castelnovo G, Murelli R, Sartori I, Galimberti CA, Tartara A: Poor sleep in adolescents: a study of 869 I 7-year-old Italian secondary school students. I Sleep Res 1997, 6:44-49.

23. Buysse DJ, Reynolds CF III, Monk TH, Berman SR, Kupfer DJ: The Pittsburgh Sleep Quality Index: a new instrument for psychiatric practice and research. Psychiatry Res 1989, 28:193-213.

24. Smyth C: The Pittsburgh Sleep Quality Index (PSQI). Insight 2000, 25:97-98.

25. Johns MW: A new method for measuring daytime sleepiness: the Epworth sleepiness scale. Sleep 1991, I4:540-545.

26. Laberge L, Gagnon C, Jean S, Mathieu J: Fatigue and daytime sleepiness rating scales in myotonic dystrophy: a study of reliability. J Neurol Neurosurg Psychiatry 2005, 76: |403-|405.

27. Taylor RR, Jason LA, Torres A: Fatigue rating scales: an empirical comparison. Psychol Med 2000, 30:849-856.

28. Chong MY, Wilkinson G: Validation of 30- and I 2-item versions of the Chinese Health Questionnaire (CHQ) in patients 
admitted for general health screening. Psychol Med 1989, 19:495-505.

29. Van RO, Mennuni G: Fatigue and sleep: the point of view of the chronobiologist. Rev Med Brux 2002, 23:A288-A293.

30. Carskadon MA: Sleep deprivation: health consequences and societal impact. Med Clin North Am 2004, 88:767-776.

31. Dement WC: Sleep extension: getting as much extra sleep as possible. Clin Sports Med 2005, 24:25I-268.

32. Dinges DF, Pack F, Williams K, Gillen KA, Powell JW, Ott GE, Aptowicz $C$, Pack Al: Cumulative sleepiness, mood disturbance, and psychomotor vigilance performance decrements during a week of sleep restricted to 4-5 hours per night. Sleep 1997, 20:267-277.

33. Horne JA: Sleep function, with particular reference to sleep deprivation. Ann Clin Res 1985, 17:199-208.

34. Lavie P, Segal S: Twenty-four-hour structure of sleepiness in morning and evening persons investigated by ultrashort sleep-wake cycle. Sleep 1989, 12:522-528.

35. Carrier J, Monk TH, Buysse DJ, Kupfer DJ: Sleep and morningness-eveningness in the 'middle' years of life (20-59 y). J Sleep Res 1997, 6:230-237.

36. Taillard J, Philip P, Bioulac B: Morningness/eveningness and the need for sleep. J Sleep Res 1999, 8:29|-295.

37. Hicks RA, Pellegrini RJ: The changing sleep habits of college students. Percept Mot Skills 1991, 72: I I06.

38. Brown FC, Buboltz WC Jr, Soper B: Development and evaluation of the Sleep Treatment and Education Program for Students (STEPS). J Am Coll Health 2006, 54:231-237.

\section{Pre-publication history}

The pre-publication history for this paper can be accessed here:

http://www.biomedcentral.com/1471-2458/9/248/pre

pub

Publish with Bio Med Central and every scientist can read your work free of charge

"BioMed Central will be the most significant development for disseminating the results of biomedical research in our lifetime."

Sir Paul Nurse, Cancer Research UK

Your research papers will be:

- available free of charge to the entire biomedical community

- peer reviewed and published immediately upon acceptance

- cited in PubMed and archived on PubMed Central

- yours - you keep the copyright

Submit your manuscript here:

http://www.biomedcentral.com/info/publishing_adv.asp
BioMedcentral 